pathway through increased phosphorylation of CHK1 on serine 345 in PEO1 olaparib-sensitive cells. Targeting PARP in combination with ATRi or CHK1i is also synergistic in olaparib-resistant OC cells. However, pCHK1 was upregulated after treatment with $\mathrm{CHK} 1 \mathrm{i}$ and PARPi/CHK1i in PEO1-OR cells. PARPi-resistance is associated with increased expression of PARP1, but not P-glycoprotein.

Conclusion* Our results show that olaparib in combination with ATRi or CHK1i prompts cell death of OC line sensitive and resistant to olaparib on the path of synthetic lethality.

This research was funded by the Polish National Science Centre, Poland (Project grant number: Sonata Bis 2019/34/E/ NZ7/00056).

\section{DIGITAL HEALTH-RELATED APP SUPPORTING PATIENTS WITH GYNAECOLOGICAL MALIGNANCIES: RESULTS OF A FEASIBILITY TRIAL}

J Wolff, I Stupin, I Sehouli*. Charité Campus Virchow Clinic, Frauenklinik, gynäkologische Onkologie, Berlin, Germany

\subsection{6/ijgc-2021-ESG0.667}

Introduction/Background* To investigate the feasibility and acceptance of the Mika health app by patients with cancer.

Methodology Prospective intra-individually controlled pilot study. Patients were randomly assigned to receive either digital psycho-oncological support in the form of the app or standard care.

Result(s)* A total of 70 patients were included, one-third of whom had high levels of psychological distress. Relative to the baseline during the 12-week period, the mean depression score decreased in the intervention group $(p=0.00078$ one-tailed, $p=0.0016$ two-tailed), without any changes in the control group. The most prevalent disease-specific side effect was fatigue, which decreased by $23.1 \%$ in the intervention group and by $31.1 \%$ in the chemotherapy subgroup $(p=0.02)$.

Conclusion* The digital app showed high acceptance and satisfaction among patients with gynaecological malignancies, regardless of age group.

\section{RESULTS OF NSGO-OV-UMB1/ENGOT-OV30 STUDY: A PHASE II STUDY OF DURVALUMAB AND OLECLUMAB IN PATIENTS WITH RELAPSED OVARIAN CANCER (OC)}

${ }^{1} \mathrm{M}$ Mirza*, ${ }^{2} \mathrm{JR}$ Henriksen, ${ }^{3} \mathrm{~J}$ Maenpaa, ${ }^{4} \mathrm{R}$ Depont Christensen, ${ }^{2} \mathrm{M}$ Waldstroem, ${ }^{5} \mathrm{~L}$ Tandaric, ${ }^{6} \mathrm{~K}$ Lindemann, ${ }^{7} \mathrm{H}$ Roed, ${ }^{3} \mathrm{~A}$ Auranen, ${ }^{8} \mathrm{~L}$ Akslen, ${ }^{9} \mathrm{M}$ Magnusson, ${ }^{9} \mathrm{~S}$ Lindberg, ${ }^{9} \mathrm{~K}$ Madsen, ${ }^{5} \mathrm{~L}$ Bjorge. ${ }^{1}$ Rigshospitalet, København, Denmark; ${ }^{2}$ Vejle University Hospital, Denmark; ${ }^{3}$ Tampere University Hospital, Finland; ${ }^{4}$ NSGO-CTU, København Ø, Denmark; ${ }^{5}$ Haukeland University Hospital, , Norway; ${ }^{6}$ Oslo university hospital, Norway; ${ }^{7}$ Rigshospitalet, Denmark; ${ }^{8}$ Haukeland University Hospital, Denmark; ${ }^{9}$ NSGO-CTU, Denmark

\subsection{6/ijgc-2021-ESG0.668}

Introduction/Background* The primary objective of this multicentre, phase II study was to evaluate the preliminary efficacy of O (3000mg, IV q2W) in combination with D (1500mg, IV, $\mathrm{q} 4 \mathrm{~W})$ in relapsed OC patients with the CD73 expression on intraepithelial inflammatory cells in the TME, defined by disease-control rate (DCR) at 16 weeks. Key eligibility criteria include: histologically confirmed OC with CD73 expression; relapsed disease; measurable disease; no prior immunotherapy; mandatory paired biopsy. 25 patients have been enrolled. Treatment was continued until disease progression, unacceptable toxicity, or withdrawal of consent. Primary endpoint is DCR; secondary endpoints include ORR, DoR, PFS, OS, safety and tolerability. Responses are also corelated to CD8 and PD-L1 positive expression in tumours. Assessment of PD-L1 $(\geq 5 \%)$ and CD8 $(\geq 5 \%)$ were performed by immunohistochemistry on archival tumour specimens. Clinical trial information: NCT03267589

Methodology The primary objective of this multicentre, phase II study was to evaluate the preliminary efficacy of $\mathrm{O}$ (3000mg, IV q2W) in combination with D (1500mg, IV, $\mathrm{q} 4 \mathrm{~W})$ in relapsed OC patients with the CD73 expression on intraepithelial inflammatory cells in the TME, defined by disease-control rate (DCR) at 16 weeks. Key eligibility criteria include: histologically confirmed OC with CD73 expression; relapsed disease; measurable disease; no prior immunotherapy; mandatory paired biopsy. 25 patients have been enrolled. Treatment was continued until disease progression, unacceptable toxicity, or withdrawal of consent. Primary endpoint is DCR; secondary endpoints include ORR, DoR, PFS, OS, safety and tolerability. Responses are also corelated to CD8 and PD-L1 positive expression in tumours. Assessment of PDL1 $(\geq 5 \%)$ and CD8 $(\geq 5 \%)$ were performed by immunohistochemistry on archival tumour specimens. Clinical trial information: NCT03267589

Result(s)* $80 \%$ of patients had received $\geq 2$ lines of therapy for relapse. DCR was 27\%, median PFS was 2.7 months and median OS was 8.4 months. $74 \%$ of patients were CD8-positive, $42 \%$ were PD-L1-positive, while $37 \%$ were both CD8 \& PD-L1-positive. Biomarker positivity was not significantly associated with better DCR $(p=0.584)$. TEAE: 1 thromboembolic event (grade 3), one neutropenia (grade 4), one cardiac arrest (grade5).

Conclusion* Combination of Oleclumab-durvalumab is feasible and demonstrate modest preliminary activity in relapsed OC. Further biomarker analysis research to predict response is ongoing.

\section{Vaginal and vulvar cancer}

\section{PRIMARY IMIQUIMOD TREATMENT VERSUS SURGERY FOR VULVAR INTRAEPITHELIAL NEOPLASIA - PITVIN STUDY. A RANDOMIZED CLINICAL TRIAL}

${ }^{1} \mathrm{G}$ Trutnovsky*, ${ }^{1} \mathrm{O}$ Reich, ${ }^{2} \mathrm{E}$ Joura, ${ }^{3} \mathrm{~A}$ Ciresa-König, ${ }^{3} \mathrm{~A}$ Widschwendter, ${ }^{4} \mathrm{C}$ Schauer ${ }^{5} \mathrm{G}$ Bogner, ${ }^{6 ; 7} \mathrm{Z}$ Jan, ${ }^{8} \mathrm{M}$ Holter, ${ }^{1} \mathrm{~K}$ Tamussino. ${ }^{1}$ Medical University of Graz, Obstetrics and Gynecology, Graz, Austria; ${ }^{2}$ Medical University of Vienna, Wien, Austria; ${ }^{3}$ Medical University of Innsbruck; ${ }^{4}$ Hospital BHB Graz; ${ }^{5}$ Medical University of Salzburg; ${ }^{6}$ Hospital Klagenfurt; ${ }^{7}$ Cancer centre Carinthia, Klinikum Klagenfurt, Klagenfurt, Austria; ${ }^{8}$ Medical University of Graz, Medical Statistics and Documentation, Graz, Austria

\subsection{6/ijgc-2021-ESG0.669}

Introduction/Background* Surgery is the standard treatment for HPV-associated high-grade vulvar intraepithelial neoplasia (HSIL/VIN), but recurrences occur in about 50\% of patients. Imiquimod, a topical immune response modifier, alters the local immune response in favour of HPV clearance and has been shown to be an effective treatment. The aim of this study was to compare the safety, effectiveness and acceptance of primary imiquimod treatment with surgical treatment of HSIL/VIN.

Methodology This was a multicentre randomised controlled trial of women with histologically confirmed HSIL/VIN. 
Exclusion criteria were clinical suspicion of microinvasion, a history of vulvar cancer, severe dermatosis, pregnancy, and any active treatment for VIN within the previous three months.

Patients were randomized to primary topical treatment or surgery at a ratio of $1: 1$ and stratified by unifocal or multifocal disease. Treatment with imiquimod was self-administered for a period of 4 months with possible extension to 6 months. Surgical treatment consisted of excision or ablation and was performed according to the standard procedures of the trial site. Clinical assessment, colposcopy, ands vulvar punch-biopsy were performed at baseline and 6 months.

Result(s)* Between June 2013 and January 2020 a total of 110 patients, aged between 19 and 82 years, were enrolled at six hospitals in Austria. 85 women (78\%) had unifocal and 24 (22\%) multifocal VIN; 21 (19\%) had received previous treatment for VIN. The primary endpoint, clinical response to treatment at 6 months, could be assessed in 107 patients. By intention to treat analysis, $39 / 56(73.6 \%)$ patients randomized to imiquimod had a complete clinical response, compared with $42 / 54(77.8 \%)$ randomized to surgery. By per-protocol analysis $37 / 46$ women (80.4\%) using imiquimod had complete clinical response which was confirmed by histology in 33 cases (89.2\%). In the per protocol surgery group 41/52 (78.8\%) women had complete clinical response. Primary or secondary surgery revealed invasive disease in five patients ( 3 pT1a, 2 pT1b). Invasive disease was not identified in any patient with per- protocol imiquimod treatment. There was no difference in treatment satisfaction between the two groups (Imiquimod: $\operatorname{median}(\min , \max )=31.0(9.0,32.0)$ and surgery: $\operatorname{median}(\min$, $\max )=31.0(12.0,32.0))$.

Conclusion* Primary treatment with imiquimod is a safe, effective and well accepted alternative to surgery in women with HSIL/VIN.

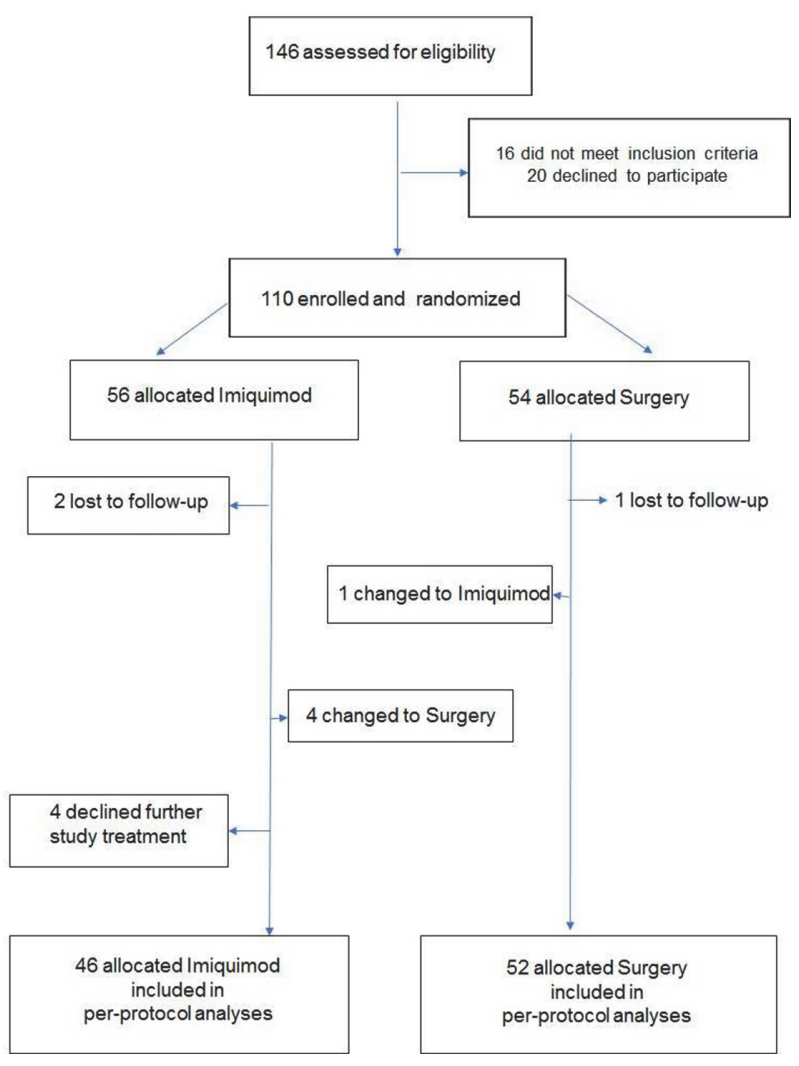

Abstract 951 Figure 1 Study population 